RESENDE, NCV; SILVA, AA; MALUF, WR; RESENDE, JTV; ZEIST, AR; GABRIEL, A. 2020. Selection of tomato lines and populations for fruit shape and resistance to tomato leafminer. Horticultura Brasileira 38: 117-125. DOI - http://doi.org/10.1590/S0102-053620200202

\title{
Selection of tomato lines and populations for fruit shape and resistance to tomato leafminer
}

\author{
Nathalia CV Resende ${ }^{1} \mathbb{D}$; Alex Antonio da Silva ${ }^{2} \mathbb{D}$; Wilson Roberto Maluf ${ }^{2} \mathbb{D}$; Juliano Tadeu V de Resende ${ }^{{ }^{\mathbb{D}}} \mathbb{D}$; \\ Andre Ricardo Zeist ${ }^{4} \mathbb{D}$; André Gabriel ${ }^{5 \mathbb{D}}$
}

${ }^{1}$ Universidade Federal de Viçosa (UFV), Viçosa-MG, Brasil; nathcvr@gmail.com; ${ }^{2}$ Universidade Federal de Lavras (UFLA), Lavras-MG, Brasil; alex.antonio.silva27@gmail.com; wrmaluf@dag.ufla.br; ${ }^{3}$ Universidade Estadual de Londrina (UEL), Londrina-PR, Brasil; jvresende@ uol.com.br; ${ }^{4}$ Universidade do Oeste Paulista, Presidente Prudente-SP, Brasil, andre.zeist@bol.com.br; ${ }^{5}$ Universidade Estadual do CentroOeste do Paraná (Unicentro), Guarapuava- PR, Brasil

\begin{abstract}
The market requirement regarding fruit type varies from region to region and it is necessary to produce cultivars with different patterns of fruit morphology. Therefore, the aim of this study was to select lines with resistance to tomato leafminer and different fruit shapes in order to meet the different market segments. Seventy-six lines and pre-selected populations were used for pest resistance together with four other susceptible controls (TOM-584, TOM-684, NC-123S, and Santa Clara) and two wild accessions (Solanum pennellii 'LA 716' and S. habrochaites var. glabratum 'PI-127826') considered resistant. The experiment consisted of tests of resistance to the tomato leafminer Tuta absoluta. The selection of lines was efficient, being obtained 33 lines or populations resistant to the tomato leafminer that comprise the market segments of the types of multilocular fruit, Santa Cruz, Saladette or Italian, as well as intermediate standards of fruits.
\end{abstract}

Keywords: Tuta absoluta, Solanum pennellii, Solanum habrochaites, acylsugar, allelochemicals, fruit shape.

\section{RESUMO}

Seleção de linhagens e populações de tomateiro para formato de fruto e resistência à traça do tomateiro

A exigência do mercado, quanto ao tipo de fruto varia de região para região, sendo necessária a produção de cultivares com diversos padrões de morfologia de fruto. Diante disso, objetivou-se com esse trabalho selecionar linhagens com resistência à traça do tomateiro e diferentes formatos de fruto, visando atender os diferentes segmentos do mercado. No experimento foram utilizadas 76 linhagens e populações pré-selecionadas, para resistência a pragas, juntamente com quatro testemunhas suscetíveis (TOM-584, TOM-684, NC$123 \mathrm{~S}$ e Santa Clara) e dois acessos selvagens de tomateiro (Solanum pennellii 'LA 716' e S. habrochaites var. glabratum 'PI-127826') considerados resistentes. $\mathrm{O}$ ensaio constituiu em testes de resistência à traça do tomateiro Tuta absoluta. A seleção das linhagens foi eficiente, sendo obtidas 33 linhagens ou populações resistentes à traça-dotomateiro que compreendem os segmentos de mercado dos tipos de fruto multilocular, Santa Cruz, saladete ou italiano, bem como frutos com padrões intermediários.

Palavras chave: Tuta absoluta, Solanum pennellii, Solanum habrochaites, acilaçúcar, zingibereno, formato de fruto.

Received on May 13, 2019; accepted on March 25, 2020

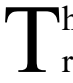
he cultivation of vegetables is related to the excessive use of agricultural pesticides, which residues persist in fruits and, therefore, increase the consumer concern. According to a research by Anvisa (2018), about 16.3\% of the collected samples of tomato were contaminated, presenting more pesticide residues than the allowed. The use of pesticides in large quantities in this crop is directly related to its susceptibility to various pests and diseases.

Among the most important pests of tomato, the tomato leafminer Tuta absoluta stands out, being considered key in tomato cultivation. Due to the frequent occurrence of these pests attacking the crop, the use of pesticides by producers has become increasingly higher. In addition, the chemical control indiscriminately used can cause damages to the environment, as well as to the health of rural workers and consumers. As a way of mitigating the use of phytosanitary products, breeding of genotypes for pest resistance has shown to be promising (Resende et al., 2009; Silva et al., 2013; Andrade et al., 2017).

Sources of resistance to pests have been found in some species of wild tomatoes, such as Solanum pennellii and Solanum habrochaites. The accessions S. pennellii 'LA 716' and $S$. habrochaites var. glabratum 'PI$127826^{\prime}$ are recognized as sources of resistance to a range of pest arthropods. The resistance factors of these species are directly related to the contents of allelochemicals present in the leaves, such as the acylsugars in $S$. pennellii and zingiberene in $S$. habrochaites. The effects of these allelochemicals are related to deleterious effects on the development of some insect phases, as 
well as to decrease its oviposition and feeding (Resende et al., 2002a; Lucini et al., 2015; Maciel et al., 2018).

The use of backcrosses for commercially acceptable lines, together with the selection oriented to obtain high levels of acylsugar and/or zingiberene, may be an efficient way to obtain commercial lines of tomatoes less dependent on the use of chemical control (Dias et al., 2013; Zanin et al., 2018). This fact would bring several benefits for the cultivation of this vegetable, such as the sustainability of the production process and the possibility of having a healthier product. In addition, it would allow a significant reduction in production costs, thus increasing profitability (Rakha et al., 2017).

Tomato breeding programs, aiming at pest resistance, have usually been based on the introgression of new resistance genes by means of interspecific crosses between the cultivated tomato and some wild species that present morphological and chemical characteristics that make them less preferred and/or damaged by arthropod pests (Resende et al., 2008; Silva, 2009, Oliveira et al., 2012; Dias et al., 2013; Neiva et al., 2013; Baier et al., 2015). Thus, a great number of lines and populations of tomato have been obtained with high contents of allelochemicals. Because elite lines used in backcrosses are considered susceptible to pests, the obtained lines and populations of tomato can segregate for different contents of allelochemicals, thus showing different resistance levels.

Therefore, it is necessary to assess the obtained lines and populations regarding their content of allelochemicals or, alternatively, directly regarding the resistance levels to pests. Thus, this study aimed to identify lines and populations of tomato with different types of fruits and higher resistance levels to the tomato leafminer Tuta absoluta.

\section{MATERIAL AND METHODS}

The experiments were conducted in a greenhouse located at the Sector of Olericulture of the Department of Agronomy of the Midwestern
State University (UNICENTRO), in Guarapuava-PR, Brazil. The city has an average altitude of $1,100 \mathrm{~m}$ and geographical coordinates of $25^{\circ} 23^{\prime} 01^{\prime \prime} \mathrm{S}$ and $51^{\circ} 29^{\prime} 46^{\prime \prime} \mathrm{W}$.

Treatments consisted of two wild accessions of tomato (Solanum pennellii 'LA 716' and S. habrochaites var. glabratum 'PI-127826'), used as resistant control treatments, and four commercial lines ('TOM-584', 'TOM684', 'NC-123S', and Santa Clara). The commercial standard lines TOM-584, TOM-684, NC-123S, and Santa Clara were used as susceptibility patterns.

These controls were tested with 76 other lines and populations of tomato for resistance to the tomato leafminer Tuta absoluta. These 76 lines and populations come from segregating populations of tomato previously selected by the breeding program of the company Hortiagro Sementes Ltda. for high leaf contents of acylsugar and/ or zingiberene. These lines with good agronomic traits were selected from interspecific crosses with 'LA-716' (high acylsugar content) and 'PI127826' (high zingiberene content), followed by several backcrosses for elite lines of cultivated tomato, including different morphologies of fruits, such as Santa Cruz, Saladette, Italian, and multilocular types; so, the lines that were used had already been evaluated for fruit shape. This evaluation consisted of the production components and postharvest. All these data were compared with the default values that determine fruit shapes.

Lines for the test, together with the controls, were sown in 128-cell polystyrene trays. The seedlings with two definitive leaves were transplanted into pots with $5 \mathrm{dm}^{3}$ substrate. At 50 days after sowing, these pots were transferred to a greenhouse previously infested with $T$. absoluta. The tomato leafminer was established from a preexisting creation, being multiplied in the greenhouse in which the experiment was carried out.

In order to assess the resistance to $T$. absoluta, leaf area, type of leaflet lesions (LL), percentage of attacked leaflets (AL), and damage to plants (DP) were assessed according to Maluf (2010b).
Only leaflets of the upper third of the plant were used for the assessments, which were previously marked with a white ribbon for further calculation of damages. After 10-day exposure to the tomato leafminer, the number of eggs was counted using a binocular microscope. Ten days after counting the damages caused by the insect to plants, the type of lesion in the leaflets, and the proportion of attacked leaflets were assessed according to a score scale proposed by Labory et al. (1999). The scale ranges from 0 to 5 , where low scores indicate lower damages (higher resistance levels). Six assessments of damages caused by the insect attack were carried out in two-day intervals. Only the average data of these six assessments were used in this study.

The experimental design was completely randomized, with the genotypes randomized in the greenhouse. Six replications of one plant were used for the controls and three replications for the other assessed genotypes, totalizing 264 plants in the greenhouse.

From the obtained data, an analysis of variance was performed and then an analysis of the means by Scott-Knott test in the statistical program $R$.

The means of treatments regarding the number of eggs per $\mathrm{cm}^{2}$, leaflet lesion (LL), percentage of attacked leaflets (AL), and damage to plants (DP) were standardized. The four standardized scores for each genotype were summed and the sum of scores was taken as a measure of the degree of resistance to the tomato leafminer. In this case, positive values were considered an indication of susceptibility to $T$. absoluta, while negative values were considered an indication of resistance.

\section{RESULTS AND DISCUSSION}

From the analysis of variance, significant differences were observed between treatments for leaflet lesions (LL), attacked leaflets [AL, (\%)], and damage to plants (DP) (Table 1). The analyses of number of eggs in this experiment were not significant. These results were similar to the ones found by Oliveira et al. (2012), there were 
Table 1. Average values of leaflet lesion (LL), attacked leaflets [AL (\%)], and damage to plants (DP) caused by Tuta absoluta on tomato plants. Guarapuava, UNICENTRO, 2018.

\begin{tabular}{|c|c|c|c|c|}
\hline Genotypes & $\begin{array}{c}\text { Average number } \\
\text { of eggs }\end{array}$ & $\mathbf{L L}$ & AL (\%) & DP \\
\hline LA-716 & 1.67 & $0.48 \mathrm{a}$ & $0.46 \mathrm{a}$ & $0.46 \mathrm{a}$ \\
\hline PI-127826 & 4.33 & $1.02 \mathrm{~b}$ & $1.04 \mathrm{a}$ & $1.21 \mathrm{~b}$ \\
\hline Santa Clara & 20.83 & $3.08 \mathrm{k}$ & $3.25 \mathrm{f}$ & $3.39 \mathrm{~g}$ \\
\hline $\mathrm{NC}-123 \mathrm{~S}$ & 16.33 & $2.91 \mathrm{j}$ & $2.94 \mathrm{~d}$ & $3.12 \mathrm{f}$ \\
\hline TOM-584 & 19.5 & $3.11 \mathrm{k}$ & $3.02 \mathrm{~d}$ & $3.58 \mathrm{~g}$ \\
\hline TOM-684 & 21.5 & 3.421 & $3.10 \mathrm{~d}$ & $3.35 \mathrm{~g}$ \\
\hline BPX-433D-09-02-106 & 14.00 & $2.98 \mathrm{j}$ & $2.83 \mathrm{~d}$ & $3.08 \mathrm{f}$ \\
\hline BPX-433D-09-02-107 & 14.33 & $2.76 \mathrm{i}$ & $2.75 \mathrm{~d}$ & $3.17 \mathrm{f}$ \\
\hline BPX-433D-09-02-111 & 9.67 & $2.41 \mathrm{~h}$ & $2.50 \mathrm{c}$ & $2.63 \mathrm{e}$ \\
\hline BPX-433D-09-02-113 & 13.00 & $2.77 \mathrm{i}$ & $2.75 \mathrm{~d}$ & $3.04 \mathrm{f}$ \\
\hline BPX-433D-12-01-101 & 13.33 & $2.50 \mathrm{~h}$ & $2.79 \mathrm{~d}$ & $2.62 \mathrm{e}$ \\
\hline BPX-433D-12-01-102 & 8.67 & $2.56 \mathrm{~h}$ & $2.67 \mathrm{~d}$ & $2.75 \mathrm{e}$ \\
\hline BPX-433D-12-01-110 & 10.67 & $2.48 \mathrm{~h}$ & $2.50 \mathrm{c}$ & $3.04 \mathrm{f}$ \\
\hline BPX-433D-12-01-111 & 12.33 & $3.07 \mathrm{k}$ & $2.79 \mathrm{~d}$ & $3.25 \mathrm{f}$ \\
\hline BPX-433D-12-09-109 & 11.67 & $2.72 \mathrm{i}$ & $2.46 \mathrm{c}$ & $2.83 \mathrm{e}$ \\
\hline BPX-433D-12-09-114 & 9.00 & $2.12 \mathrm{f}$ & $2.33 \mathrm{c}$ & $2.37 \mathrm{~d}$ \\
\hline BPX-437E-02-01-02-03 & 10.33 & $2.43 \mathrm{~h}$ & $2.50 \mathrm{~d}$ & $2.50 \mathrm{~d}$ \\
\hline BPX-437E-02-01-03-01 & 6.00 & $1.68 \mathrm{c}$ & $2.08 \mathrm{a}$ & $1.83 \mathrm{c}$ \\
\hline BPX-437E-02-01-03-02 & 8.00 & $2.01 \mathrm{e}$ & $2.21 \mathrm{~b}$ & $1.96 \mathrm{c}$ \\
\hline BPX-437E-02-01-03-07 & 11.00 & $1.92 \mathrm{e}$ & $2.00 \mathrm{a}$ & $1.92 \mathrm{c}$ \\
\hline BPX-437E-09-03-02-03 & 12.33 & $2.26 \mathrm{~g}$ & $2.42 \mathrm{c}$ & $2.75 \mathrm{e}$ \\
\hline BPX-437E-09-03-02-04 & 14.00 & $2.65 \mathrm{i}$ & $2.96 \mathrm{~d}$ & $2.92 \mathrm{e}$ \\
\hline BPX-437E-09-03-04-03 & 11.00 & $2.76 \mathrm{i}$ & $2.29 \mathrm{c}$ & $3.29 \mathrm{~g}$ \\
\hline BPX-437E-09-03-04-04 & 10.00 & $2.29 \mathrm{~g}$ & $2.75 \mathrm{~d}$ & $2.67 \mathrm{e}$ \\
\hline BPX-437E-09-03-04-09 & 7.33 & $2.01 \mathrm{e}$ & $2.21 \mathrm{~b}$ & $2.04 \mathrm{c}$ \\
\hline BPX-437E-09-03-04-10 & 9.67 & $1.96 \mathrm{e}$ & $2.50 \mathrm{c}$ & $2.29 \mathrm{~d}$ \\
\hline BPX-443D-03-02-102 & 8.33 & $1.96 \mathrm{e}$ & $2.67 \mathrm{~d}$ & $2.00 \mathrm{c}$ \\
\hline BPX-443D-03-02-113 & 10.33 & $2.04 \mathrm{e}$ & $2.13 \mathrm{~b}$ & $1.92 \mathrm{c}$ \\
\hline BPX-443D-05-06-101 & 7.67 & $2.31 \mathrm{~g}$ & $2.08 \mathrm{~b}$ & $2.54 \mathrm{e}$ \\
\hline BPX-443D-05-06-108 & 14.67 & $2.77 \mathrm{i}$ & $2.75 \mathrm{~d}$ & $3.04 \mathrm{f}$ \\
\hline BPX-443D-05-06-111 & 11.00 & $2.14 \mathrm{f}$ & $2.75 \mathrm{~d}$ & $2.58 \mathrm{e}$ \\
\hline BPX-443D-05-06-112 & 9.67 & $2.05 \mathrm{e}$ & $2.46 \mathrm{c}$ & $2.33 \mathrm{~d}$ \\
\hline BPX-443D-05-07-104 & 9.67 & $2.37 \mathrm{~h}$ & $2.42 \mathrm{c}$ & $2.58 \mathrm{e}$ \\
\hline BPX-443D-05-07-105 & 9.00 & $2.84 \mathrm{j}$ & $3.04 \mathrm{~d}$ & $3.50 \mathrm{~g}$ \\
\hline BPX-443D-05-07-111 & 9.67 & $2.73 \mathrm{i}$ & $3.13 \mathrm{e}$ & $3.12 \mathrm{f}$ \\
\hline BPX-455D-10 & 7.00 & $2.23 \mathrm{~g}$ & $2.67 \mathrm{~d}$ & $2.54 \mathrm{e}$ \\
\hline BPX-455D-18 & 7.67 & $2.05 \mathrm{e}$ & $2.50 \mathrm{~d}$ & $2.25 \mathrm{~d}$ \\
\hline BPX-455D-21 & 15.00 & $2.44 \mathrm{~h}$ & $2.50 \mathrm{~d}$ & $2.62 \mathrm{e}$ \\
\hline BPX-455D-34 & 10.33 & $1.97 \mathrm{e}$ & $2.83 \mathrm{~d}$ & $2.37 \mathrm{~d}$ \\
\hline BPX-455D-36 & 8.67 & $2.08 \mathrm{f}$ & $2.21 \mathrm{c}$ & $2.46 \mathrm{~d}$ \\
\hline BPX-465D-34-08-04-103 & 9.33 & $2.34 \mathrm{~g}$ & $2.46 \mathrm{c}$ & $2.25 \mathrm{~d}$ \\
\hline BPX-465D-34-08-04-104 & 8.67 & $1.83 \mathrm{~d}$ & $2.21 \mathrm{~b}$ & $2.29 \mathrm{~d}$ \\
\hline
\end{tabular}


Table 1. continuation..

\begin{tabular}{|c|c|c|c|c|}
\hline Genotypes & $\begin{array}{c}\text { Average number } \\
\text { of eggs }\end{array}$ & $\mathbf{L L}$ & AL (\%) & DP \\
\hline BPX-465D-34-08-04-105 & 13.33 & $2.50 \mathrm{~h}$ & $2.54 \mathrm{~d}$ & $2.00 \mathrm{c}$ \\
\hline BPX-465D-34-08-04-106 & 9.00 & $1.78 \mathrm{~d}$ & $2.25 \mathrm{c}$ & $1.71 \mathrm{c}$ \\
\hline BPX-465D-34-08-04-110 & 8.00 & $1.97 \mathrm{e}$ & $2.17 \mathrm{~b}$ & $2.04 \mathrm{c}$ \\
\hline BPX-465D-34-08-04-111 & 17.00 & $2.18 \mathrm{f}$ & $2.58 \mathrm{~d}$ & $2.33 \mathrm{~d}$ \\
\hline BPX-465D-34-08-04-113 & 8.00 & $2.30 \mathrm{~g}$ & $2.67 \mathrm{~d}$ & $2.37 \mathrm{~d}$ \\
\hline BPX-465D-34-08-04-114 & 11.00 & $2.16 \mathrm{f}$ & $2.42 \mathrm{c}$ & $2.25 \mathrm{~d}$ \\
\hline BPX-465D-34-08-07-101 & 13.00 & $2.26 \mathrm{~g}$ & $2.33 \mathrm{c}$ & $2.12 \mathrm{~d}$ \\
\hline BPX-465D-34-08-07-112 & 12.00 & $2.65 \mathrm{i}$ & $2.67 \mathrm{~d}$ & $2.46 \mathrm{~d}$ \\
\hline BPX-465D-34-08-07-113 & 14.67 & $2.33 \mathrm{~g}$ & $2.54 \mathrm{~d}$ & $2.29 \mathrm{~d}$ \\
\hline BPX-465D-34-08-11-103 & 10.67 & $2.51 \mathrm{~h}$ & $2.92 \mathrm{~d}$ & $2.75 \mathrm{e}$ \\
\hline BPX-465D-34-08-11-106 & 14.00 & $2.00 \mathrm{e}$ & $2.46 \mathrm{c}$ & $2.00 \mathrm{c}$ \\
\hline BPX-465D-34-08-11-111 & 15.33 & $2.22 \mathrm{~g}$ & $2.67 \mathrm{~d}$ & $2.21 \mathrm{~d}$ \\
\hline BPX-465D-34-08-16-101 & 11.33 & $2.30 \mathrm{~g}$ & $2.54 \mathrm{~d}$ & $2.25 \mathrm{~d}$ \\
\hline BPX-465D-34-08-16-102 & 8.00 & $2.08 \mathrm{f}$ & $2.50 \mathrm{~d}$ & $1.92 \mathrm{c}$ \\
\hline BPX-465D-34-08-16-104 & 8.33 & $1.90 \mathrm{e}$ & $2.62 \mathrm{~d}$ & $1.96 \mathrm{c}$ \\
\hline BPX-465D-34-08-16-111 & 11.67 & $2.40 \mathrm{~h}$ & $2.25 \mathrm{c}$ & $2.29 \mathrm{~d}$ \\
\hline BPX-465D-34-08-26-106 & 7.33 & $1.75 \mathrm{~d}$ & $2.17 \mathrm{~b}$ & $1.71 \mathrm{c}$ \\
\hline BPX-465D-34-08-26-111 & 8.67 & $1.93 \mathrm{e}$ & $2.22 \mathrm{~b}$ & $1.83 \mathrm{c}$ \\
\hline BPX-466D-118-03-01-103 & 8.67 & $1.78 \mathrm{~d}$ & $1.87 \mathrm{a}$ & $1.79 \mathrm{c}$ \\
\hline BPX-466D-118-03-07-114 & 7.33 & $2.00 \mathrm{e}$ & $2.00 \mathrm{a}$ & $1.92 \mathrm{c}$ \\
\hline BPX-466D-174-01-05-106 & 9.67 & $2.44 \mathrm{~h}$ & $2.08 \mathrm{~b}$ & $2.33 \mathrm{~d}$ \\
\hline BPX-466D-174-01-05-108 & 9.67 & $2.15 \mathrm{f}$ & $2.25 \mathrm{c}$ & $1.96 \mathrm{c}$ \\
\hline BPX-466D-174-02-07-105 & 9.67 & $2.26 \mathrm{~g}$ & $2.50 \mathrm{~d}$ & $2.42 \mathrm{~d}$ \\
\hline BPX-466D-174-02-07-111 & 9.67 & $1.83 \mathrm{~d}$ & $2.38 \mathrm{c}$ & $1.92 \mathrm{c}$ \\
\hline BPX-466D-221-05-01-104 & 9.33 & $1.93 \mathrm{e}$ & $2.46 \mathrm{c}$ & $2.33 \mathrm{~d}$ \\
\hline BPX-466D-221-05-01-105 & 8.33 & $2.32 \mathrm{~g}$ & $2.46 \mathrm{c}$ & $2.21 \mathrm{~d}$ \\
\hline BPX-466D-221-05-01-112 & 11.00 & $2.23 \mathrm{~g}$ & $2.62 \mathrm{~d}$ & $2.25 \mathrm{~d}$ \\
\hline BPX-513A-01 & 11.33 & $2.03 \mathrm{e}$ & $2.25 \mathrm{c}$ & $2.00 \mathrm{c}$ \\
\hline BPX-513A-03 & 8.33 & $2.08 \mathrm{f}$ & $2.37 \mathrm{c}$ & $2.00 \mathrm{c}$ \\
\hline BPX-513A-20 & 7.00 & $1.65 \mathrm{c}$ & $2.17 \mathrm{~b}$ & $1.71 \mathrm{c}$ \\
\hline BPX-514A-07 & 12.00 & $2.11 \mathrm{f}$ & $2.29 \mathrm{c}$ & $2.29 \mathrm{~d}$ \\
\hline BPX-514A-09 & 10.00 & $2.26 \mathrm{~g}$ & $2.25 \mathrm{c}$ & $2.17 \mathrm{~d}$ \\
\hline BPX-514A-15 & 7.66 & $2.19 \mathrm{f}$ & $2.25 \mathrm{c}$ & $2.29 \mathrm{~d}$ \\
\hline BPX-514A-17 & 7.33 & $2.19 \mathrm{f}$ & $2.83 \mathrm{~d}$ & $2.46 \mathrm{~d}$ \\
\hline BPX-515A-03 & 5.67 & $1.83 \mathrm{~d}$ & $2.21 \mathrm{c}$ & $1.92 \mathrm{c}$ \\
\hline BPX-515A-04 & 12.67 & $2.10 \mathrm{f}$ & $2.25 \mathrm{c}$ & $2.00 \mathrm{c}$ \\
\hline BPX-515A-07 & 10.67 & $2.08 \mathrm{f}$ & $2.37 \mathrm{c}$ & $2.21 \mathrm{~d}$ \\
\hline BPX-515A-17 & 6.33 & $1.86 \mathrm{~d}$ & $2.21 \mathrm{~b}$ & $1.83 \mathrm{c}$ \\
\hline BPX-516A-04 & 10.33 & $2.14 \mathrm{f}$ & $2.25 \mathrm{c}$ & $2.37 \mathrm{~d}$ \\
\hline BPX-516A-10 & 6.33 & $1.97 \mathrm{e}$ & $2.00 \mathrm{a}$ & $1.79 \mathrm{c}$ \\
\hline
\end{tabular}

Means followed by same letters in the column belong to the same group by Scott-Knott test, $5 \%$ probability. Susceptibility of the genotypes decreases from letters ' $a$ ' to ' 1 '. LL: values range from 0 to 5 , where low scores indicate lower damages (higher resistance levels). 
Table 2. Standardized (std) means of genotypes from the coefficient of variation. Guarapuava, UNICENTRO, 2018.

\begin{tabular}{|c|c|c|c|c|c|}
\hline Genotypes & Eggs (std) & DP (std) & AL $(\%$, std $)$ & LL (std) & Sum (std) \\
\hline LA-716 & -2.60 & -3.82 & -4.02 & -4.50 & -14.94 \\
\hline PI-127826 & -1.81 & -2.46 & -2.61 & -2.99 & -9.88 \\
\hline TOM-584 & 2.68 & 1.39 & 1.13 & 0.53 & 5.73 \\
\hline NC-123S & 1.74 & 0.94 & 0.66 & 0.78 & 4.12 \\
\hline Santa Clara & 3.07 & 1.17 & 0.9 & 1.03 & 6.17 \\
\hline TOM-684 & 3.27 & 1.17 & 1.13 & 1.28 & 6.85 \\
\hline BPX-433D-09-02-111 & -0.23 & -0.19 & 0.2 & -0.48 & -0.71 \\
\hline BPX-433D-12-09-114 & -0.43 & -0.19 & -0.74 & -0.48 & -1.84 \\
\hline BPX-437E-02-01-03-01 & -1.32 & -1.1 & -1.21 & -0.98 & -4.61 \\
\hline BPX-437E-02-01-03-02 & -0.73 & -0.65 & -0.74 & -0.48 & -2.59 \\
\hline BPX-437E-02-01-03-07 & 0.16 & -0.65 & -0.27 & -0.98 & -1.74 \\
\hline BPX-437E-09-03-04-09 & -0.92 & -1.1 & -0.74 & -0.48 & -3.24 \\
\hline BPX-443D-03-02-113 & -0.04 & -0.65 & -0.27 & 0.02 & -0.93 \\
\hline BPX-443D-05-06-101 & -0.83 & -0.19 & -0.74 & -1.48 & -3.24 \\
\hline BPX-443D-05-06-112 & -0.23 & -0.65 & -0.74 & 0.02 & -1.60 \\
\hline BPX-455D-10 & -1.02 & -0.19 & -0.27 & 0.53 & -0.96 \\
\hline BPX-455D-18 & -0.83 & -0.65 & -0.74 & -0.48 & -2.69 \\
\hline BPX-455D-36 & -0.53 & 0.26 & -0.27 & -0.98 & -1.52 \\
\hline BPX-465D-34-08-04-103 & -0.33 & -0.19 & -0.27 & -0.48 & -1.28 \\
\hline BPX-465D-34-08-04-104 & -0.53 & -0.19 & -1.21 & -0.48 & -2.41 \\
\hline BPX-465D-34-08-04-106 & -0.43 & -1.55 & -0.74 & -0.98 & -3.70 \\
\hline BPX-465D-34-08-04-110 & -0.73 & -0.65 & -0.74 & -0.98 & -3.09 \\
\hline BPX-465D-34-08-04-113 & -0.73 & -0.65 & -0.27 & 0.02 & -1.62 \\
\hline BPX-465D-34-08-04-114 & 0.16 & -0.19 & -0.74 & -0.48 & -1.25 \\
\hline BPX-465D-34-08-16-102 & -0.73 & -1.10 & -0.27 & 0.53 & -1.57 \\
\hline BPX-465D-34-08-16-104 & -0.63 & -1.10 & -1.21 & -0.48 & -3.41 \\
\hline BPX-465D-34-08-26-106 & -0.92 & -1.10 & -1.21 & 0.02 & -3.21 \\
\hline BPX-465D-34-08-26-111 & -0.53 & -1.10 & -0.74 & -0.48 & -2.85 \\
\hline BPX-466D-118-03-01-103 & -0.53 & -1.10 & -0.74 & -1.99 & -4.36 \\
\hline BPX-466D-118-03-07-114 & -0.92 & -0.65 & -0.27 & -1.48 & -3.33 \\
\hline BPX-466D-174-01-05-108 & -0.23 & -0.65 & -0.27 & -0.48 & -1.63 \\
\hline BPX-466D-174-02-07-111 & -0.23 & -1.10 & -1.21 & 0.02 & -2.52 \\
\hline BPX-513A-03 & -0.63 & -0.65 & -0.27 & 0.02 & -1.52 \\
\hline BPX-513A-20 & -1.02 & -1.55 & -1.68 & -0.48 & -4.73 \\
\hline BPX-514A-15 & -0.83 & -0.65 & -0.27 & -0.48 & -2.22 \\
\hline BPX-515A-03 & -1.42 & -1.10 & -1.21 & -0.98 & -4.71 \\
\hline BPX-515A-07 & 0.06 & -0.19 & -0.27 & -0.48 & -0.88 \\
\hline BPX-515A-17 & -1.22 & -0.65 & -0.74 & -0.98 & -3.59 \\
\hline BPX-516A-10 & -1.22 & -1.10 & -0.74 & -1.48 & -4.54 \\
\hline BPX-433D-09-02-106 & 1.05 & 1.62 & 2.07 & 1.53 & 6.27 \\
\hline BPX-433D-09-02-107 & 1.15 & 1.62 & 1.60 & 0.53 & 4.89 \\
\hline BPX-433D-09-02-113 & 0.75 & 0.71 & 1.13 & 0.53 & 3.13 \\
\hline BPX-433D-12-01-101 & 0.85 & 0.71 & 1.13 & 1.03 & 3.73 \\
\hline
\end{tabular}


Table 2. continuation...

\begin{tabular}{|c|c|c|c|c|c|}
\hline Genotypes & Eggs (std) & DP (std) & $\operatorname{AL}(\%$, std $)$ & LL (std) & Sum (std) \\
\hline BPX-433D-12-01-102 & -0.53 & 0.26 & 0.20 & 0.02 & -0.05 \\
\hline BPX-433D-12-01-110 & 0.06 & 1.62 & 0.20 & -0.48 & 1.40 \\
\hline BPX-433D-12-01-111 & 0.56 & 1.62 & 1.60 & 0.53 & 4.30 \\
\hline BPX-433D-12-09-109 & 0.36 & 0.71 & 1.60 & 0.02 & 2.70 \\
\hline BPX-437E-02-01-02-03 & -0.04 & 1.17 & 1.13 & 0.02 & 2.29 \\
\hline BPX-437E-09-03-02-03 & 0.56 & 0.71 & 0.20 & 0.53 & 1.99 \\
\hline BPX-437E-09-03-02-04 & 1.05 & 1.17 & 1.60 & 1.53 & 5.35 \\
\hline BPX-437E-09-03-04-03 & 0.16 & 2.07 & 1.60 & 0.02 & 3.86 \\
\hline BPX-437E-09-03-04-04 & -0.14 & 1.62 & 0.66 & 1.03 & 3.18 \\
\hline BPX-437E-09-03-04-10 & -0.23 & 0.26 & -0.27 & 1.03 & 0.78 \\
\hline BPX-443D-03-02-102 & -0.63 & -0.19 & -0.27 & 1.03 & -0.06 \\
\hline BPX-443D-05-06-108 & 1.25 & 1.17 & 1.13 & 1.03 & 4.58 \\
\hline BPX-443D-05-06-111 & 0.16 & 0.71 & 0.20 & 1.03 & 2.10 \\
\hline BPX-443D-05-07-104 & -0.23 & 0.26 & 0.20 & 0.02 & 0.25 \\
\hline BPX-443D-05-07-105 & -0.43 & 1.62 & 1.60 & 1.53 & 4.32 \\
\hline BPX-443D-05-07-111 & -0.23 & 1.62 & 1.13 & 1.53 & 4.05 \\
\hline BPX-455D-21 & 1.34 & 0.71 & 0.66 & 0.02 & 2.75 \\
\hline BPX-455D-34 & -0.04 & -0.19 & -0.74 & 0.53 & -0.44 \\
\hline BPX-465D-34-08-04-105 & 0.85 & -0.19 & 1.13 & 0.53 & 2.32 \\
\hline BPX-465D-34-08-04-111 & 1.94 & 0.26 & 0.20 & 1.03 & 3.42 \\
\hline BPX-465D-34-08-07-101 & 0.75 & -0.19 & 0.20 & 0.02 & 0.78 \\
\hline BPX-465D-34-08-07-112 & 0.46 & 0.26 & 1.60 & 1.03 & 3.35 \\
\hline BPX-465D-34-08-07-113 & 1.25 & 0.26 & 0.20 & 0.02 & 1.73 \\
\hline BPX-465D-34-08-11-103 & 0.06 & 0.71 & 0.66 & 1.53 & 2.97 \\
\hline BPX-465D-34-08-11-106 & 1.05 & -0.19 & -0.27 & 0.53 & 1.11 \\
\hline BPX-465D-34-08-11-111 & 1.44 & 0.26 & 0.20 & 1.03 & 2.93 \\
\hline BPX-465D-34-08-16-101 & 0.26 & -0.19 & 0.2 & 0.02 & 0.29 \\
\hline BPX-465D-34-08-16-111 & 0.36 & 0.26 & 0.66 & -0.48 & 0.8 \\
\hline BPX-466D-174-01-05-106 & -0.23 & 0.26 & 0.66 & -0.48 & 0.21 \\
\hline BPX-466D-174-02-07-105 & -0.23 & 0.26 & 0.20 & 0.53 & 0.75 \\
\hline BPX-466D-221-05-01-104 & -0.33 & 0.71 & -0.74 & 0.53 & 0.17 \\
\hline BPX-466D-221-05-01-105 & -0.63 & -0.19 & 0.66 & 0.53 & 0.37 \\
\hline BPX-466D-221-05-01-112 & 0.16 & 0.26 & 0.20 & 1.03 & 1.65 \\
\hline BPX-513A-01 & 0.26 & -0.19 & 0.20 & 0.02 & 0.29 \\
\hline BPX-514A-07 & 0.46 & 0.26 & -0.27 & -0.48 & -0.03 \\
\hline BPX-514A-09 & -0.14 & -0.19 & 0.20 & 0.02 & -0.11 \\
\hline BPX-514A-17 & -0.92 & 0.71 & -0.27 & 1.03 & 0.55 \\
\hline BPX-515A-04 & 0.65 & -0.19 & 0.20 & 0.02 & 0.68 \\
\hline BPX-516A-04 & -0.04 & 0.26 & 0.20 & 0.02 & 0.44 \\
\hline General mean & 10.46 & 3.48 & 3.65 & 3.53 & \\
\hline Standard deviation & 3.38 & 0.74 & 0.66 & 0.71 & \\
\hline
\end{tabular}

Standardized data from the coefficient of variation. = negative values indicate higher resistance levels. DP: damage to plants; AL: percentage of atacket leaflets; LL: leaflet lesion. 
significant differences for severity of damage to the plants, injuries to the leaflets, and percentage of leaflets attacked indicating a reduction by the presence of the allelochemicals. However, he also found a significant difference for number of eggs, which was not significant in this experiment. On the other hand, Dias et al. (2019) researching resistance of processing tomato genotypes to leafminer (Tuta absoluta), using the same method used in the current study did not find significant differences for number of eggs.

A significant difference was observed between the resistant accessions and commercial controls (Table 1). Similarly, the resistance of many of the tested lines and populations, which presented averages close to that of the resistant controls, with high contents of allelochemicals (Table 1), was evident. In this context, the genotypes BPX437E-02-01-03-01 and BPX-513A-20 stood out, since they demonstrated to be superior and had a mean closer to the resistant parental for all analyzed traits. Dias et al. (2019), evaluating the resistance of improved tomato genotypes, rich in allelochemicals, found significant differences between the commercial control and the evaluated genotypes and the similarity with the wild species content of allelochemicals.

In addition, some lines and populations, even resulting from the selection process in previous generations for high acylsugar content, obtained a mean closer to the susceptible controls. This trait was reported to be controlled by a larger incompletely recessive gene and a minor additive gene (Resende et al., 2002b; Gonçalves et al., 2007). Baier et al. (2015), studying genetic control to acylsugar, found a number of genes estimated of 0.69 under the assumption that a single genetic locus is responsible for the genetic control of the acylsugar content in the interspecific $S$. lycopersicum and $S$. pennellii cross. They also found that an additive dominant model revealed the presence of a recessive locus in $S$. pennellii with incomplete dominance that is responsible for the high acylsugar content. However, studies (Maluf et al., 2010a) indicated a predominantly additive gene action. Thus, the selection in previous generations would have led to obtaining plants still heterozygous for the trait, which would explain the existence of susceptible lines in later generations.

Among the 76 lines, 33 were selected based on the sum of scores of the standardized data (Table 2), which had means closer to the resistant controls, i.e. they had resistance to the tomato leafminer. These results show that the heritability of resistance is high, which makes possible to associate, in improved lines, commercial quality and good levels of resistance to arthropod pests. Baier et al. (2015) confirmed that

Table 3. Selected lines of tomato with respective fruit shapes. Guarapuava, UNICENTRO, 2018.

\begin{tabular}{|c|c|c|}
\hline Control & $\begin{array}{c}\text { Sum of standardized } \\
\text { scores }{ }^{1}\end{array}$ & Fruit shape \\
\hline LA-716 & -14.94 & Wild \\
\hline PI-127826 & -9.88 & Wild \\
\hline Santa Clara & 5.73 & Santa Cruz \\
\hline NC-123S & 4.12 & Multilocular \\
\hline TOM-584 & 6.17 & Santa Cruz \\
\hline \multicolumn{3}{|l|}{ Selected lines } \\
\hline BPX-443D-03-02-113 & -0.93 & Santa Cruz \\
\hline BPX-443D-05-06-101 & -3.24 & Multilocular \\
\hline BPX-443D-05-06-112 & -1.60 & Multilocular \\
\hline BPX-455D-10 & -0.96 & Santa Cruz \\
\hline BPX-455D-18 & -2.69 & Saladette \\
\hline BPX-455D-36 & -1.52 & Saladette \\
\hline BPX-465D-34-08-04-103 & -1.28 & Saladette \\
\hline BPX-465D-34-08-04-104 & -2.41 & Saladette \\
\hline BPX-465D-34-08-04-106 & -3.70 & Saladette \\
\hline BPX-465D-34-08-04-110 & -3.09 & Saladette \\
\hline BPX-465D-34-08-04-113 & -1.62 & Saladette \\
\hline BPX-465D-34-08-04-114 & -1.25 & Saladette \\
\hline BPX-465D-34-08-16-102 & -1.57 & Santa Cruz \\
\hline BPX-465D-34-08-16-104 & -3.41 & Saladette \\
\hline BPX-465D-34-08-26-106 & -3.21 & Saladette \\
\hline BPX-465D-34-08-26-111 & -2.85 & Saladette \\
\hline BPX-466D-118-03-01-103 & -4.36 & Multilocular \\
\hline BPX-466D-118-03-07-114 & -3.33 & Saladette \\
\hline BPX-466D-174-01-05-108 & -1.63 & Saladette \\
\hline BPX-466D-174-02-07-111 & -2.52 & Santa Cruz+ Saladette \\
\hline BPX-513A-03 & -1.52 & Santa Cruz+ Saladette \\
\hline BPX-513A-20 & -4.73 & Notrated \\
\hline BPX-514A-15 & -2.22 & Italiano \\
\hline BPX-515A-03 & -4.71 & Santa Cruz a Italiano \\
\hline BPX-515A-07 & -0.88 & Notrated \\
\hline BPX-515A-17 & -3.59 & Notrated \\
\hline BPX-516A-10 & -4.54 & Notrated \\
\hline
\end{tabular}

${ }^{1}$ Sum of standardized scores $=$ negative values indicate higher resistance levels. 
the estimated value of the broad sense heritability for acylsugar content was $81.85 \%$, and Lima et al. (2015), found that for high zingiberene content the heritability was high too (81.9\%).

The selected lines have different fruit shapes (Table 3). The assessments to define the fruit shape of these lines were carried out in previous experiments. In this study we aimed to select fruits of the multilocular, Santa Cruz, Saladette shape or, shapes close to them. These results will allow continuing the breeding program, with commercial hybrids with different types of fruit, meeting the demands of the different market segments.

So, with this experiment we could identify genotypes (lines and populations) of tomato plants with good resistance levels to T. absoluta, mediated by exudates from allelochemicals. Lima et al. (2015) found that the selection for high zingiberene content is an efficient way to obtain plants presenting resistance to the tomato leafminer. Lucini et al. (2015) also studied the resistance mechanisms presented by $F_{2}$ genotypes with contrasting acylsugar contents from a crossing between $S$. lycopersicum and $S$. pennellii accession 'LA-716'. By means of choice and nochoice tests, the presence of resistance mechanisms by antixenosis and antibiosis in $\mathrm{F}_{2}$ genotypes selected for high acylsugar contents, was identified when submitted to the presence of the tomato leafminer. All these studies confirm the efficiency of the selection through the allelochemicals levels. The genotypes obtained in this study have fruits with a shape for the referring groups Santa Cruz, Saladette, Italian, and multilocular, in addition to some fruits with an intermediate shape. This way we could relate resistance with specific fruit shape that the consumer market requires.

The genotypes with different fruit formats presented resistance to tomato leafminer, mediated by allelochemicals. Thus, enabling to obtain lines with different backgrounds, available for obtaining hybrids.

\section{ACKNOWLEDGEMENTS}

The authors thank Fundação Araucária de Apoio ao Desenvolvimento Científico e Tecnológico do Paraná (Foundation for Support on Scientific and Technological Development of Paraná State) for financial support, the Conselho Nacional de Desenvolvimento Científico e Tecnológico (CNPq) (National Council for Scientific and Technological Development) for the Research Incentive scholarships granted and Coordenação de Aperfeiçoamento de Pessoal de Nível Superior (CAPES) (Coordination for Improvement of Higher Education Personnel) for Master scholarship granted.

\section{REFERENCES}

ANDRADE, MC; SILVA, AA; NEIVA, IP; OLIVEIRA, IRC; CASTRO, EM; FRANCIS, DM; MALUF, WR. 2017. Inheritance of type IV glandular trichome density and its association with whitefly resistance from Solanum galapagense accession LA1401. Euphytica 213 , n.2.

ANVISA, 2018. 'ANVISA: Agrotóxicos no Brasil'. Available http://www.anvisa.gov.br. AccessOctober 7, 2019.

BAIER, JE; RESENDE, JTV; FARIA, MV; SCHWARZ, K; MEERT, L. 2015. Indirect selection of industrial tomato genotypes resistant to spider mite Tetranychus urticae. Genetics and Molecular Research 14:244-252.

DIAS, DM; RESENDE, JTV; FARIA, MV; CAMARGO, LKP; LIMA, IP. 2013. Selection of processing tomato genotypes with high acyl sugar content that are resistant to the tomato pinworm. Genetics and Molecular Research 12: 381-389.

DIAS, DM; RESENDE, JTV; ZEIST, AR; GABRIEL, A; SANTOS, MH; RESENDE, NCV. 2019. Resistance of processing tomato genotypes to leafminer (Tuta absoluta). Horticultura Brasileira 37: 40-46.

GONÇALVES, LD; MALUF, WR; CARDOSO, MG; GOMES, LAA. 2007. Herança de acilaçúcares em genótipos de tomateiro provenientes de cruzamento interespecífico. Pesquisa Agropecuária Brasileira 42: 699705.

LABORY, CRG; SANTA-CECILIA, LVC; MALUF, WR; CARDOSO, MG; BEARZOTTI, E; SOUZA, JC. 1999. Seleção indireta para teor de 2-tridecanona em tomateiros segregantes e sua relação com a resistência à traça-do-tomateiro. Pesquisa Agropecuária Brasileira 34: 733-740.
LIMA, IP; RESENDE, JTV; OLIVEIRA, JRF; FARIA, MV; RESENDE, NCV; LIMA FILHO, RB. 2015. Indirect selection of industrial tomato genotypes rich in zingiberene and resistant to Tuta absoluta Meyrick. Genetics and Molecular Research 14: 1508115089 .

LUCINI, T; FARIA, MV; ROHDE, C; RESENDE, JTV; OLIVEIRA, JRF. 2015. Acylsugar and the role of tricomes in tomato genotypes resistance to Tetranychus urticae. ArthopodPlant Interactions 9: 45-53.

MACIEL, GM; MARQUEZ, GR; SILVA EC; ANDALÓ, V; BELLOTI, IF. 2018. Tomato genotypes with determinate growth and high acylsugar content presenting resistance to spider mite. Crop Breeding and Applied Biotechnology 18: 1-8.

MALUF, WR; MACIEL, GM; GOMES, LAA; CARDOSO, MG; GONÇALVES, LD; SILVA, EC; KNAPP, M. 2010a. Broad-spectrum arthropod resistance in hybrids between highand low-acylsugar tomato lines. Crop Science 50: 439-450.

MALUF, WR; SILVA, VF; CARDOSO, MG; GOMES, LAA; NETO, ACG; MACIEL, GM; NÍZIO, DAC. 2010b. Resistance to the South American tomato pinworm Tuta absoluta in high acylsugar and/or high zingiberene tomato genotypes. Euphytica 176: 113-123.

NEIVA, IP; ANDRADE JÚNIOR, VC; MALUF, WR; OLIVEIRA, CM; MACIEL, GM. 2013. Role of allelochemicals and trichome density in the resistance of tomato to whitefly. Ciencia e Agrotecnologia 37: 61-67.

OLIVEIRA, CM; ANDRADE JUNIOR, VC; MALUF, WR; NEIVA, IP; MACIEL, GM. 2012. Resistance of tomato strains to the moth Tuta absoluta imparted by allelochemicals and trichome density. Ciência e Agrotecnologia 36: 45-52.

RAKHA, M; BOUBA, I; RAMASAMY, S; REGNARD, JL; HANSON, P. 2017. Evaluation of wild tomato accessions (Solanum spp.) for resistance to two-spotted spidermite (Tetranychus urticae) based on trichome type and acylsugar content. Genetics Resources and Crop Evolution 64: 1011-1022.

RESENDE, JTV; CARDOSO, MG; MALUF, WR; SANTOS, CD; GONÇALVES, LD; RESENDE, LV; NAVES FO. 2002a. Método colorimétrico para quantificação de acilaçúcar em genótipos de tomateiro. Ciência e Agrotecnologia 26: 1204-1208

RESENDE, JTV; MALUF, WR; CARDOSO, MG; FARIA, MV; GONÇALVES, LD; NASCIMENTO, IR. 2008. Resistance of tomato genotypes with high level of acylsugars to Tetranychus evansi Baker \& Pritchard. Scientia Agricola 65: 31-35.

RESENDE, JTV; MALUF, WR; CARDOSO, MG; GONÇALVES, LD; FARIA, MV; NASCIMENTO, IR. 2009. Resistance of tomato genotypes to the silverleaf whitefly mediated by acylsugars. Horticultura brasileira 27: 345-348.

RESENDE, JTV; MALUF, WR; CARDOSO, 
MG; NELSON, DL; FARIA, MV. 2002 b. Inheritance of acylsugar contents in tomatoes derived from an interspecific cross with the wild tomato Lycopersicon pennellii and their effect on spider mite repellence. Genetics and Molecular Research 1: 106-116.

SILVA, VF. 2009. Resistência mediada por aleloquímicos de genótipos de tomateiro à mosca-branca e ao ácaro-rajado. Pesquisa Agropecuária Brasileira 44: 1262-1269.

SILVA, AA; MALUF, WR; MORAES, JC; ALVARENGA, R; MARIA, E; COSTA R. 2013. Resistência a Myzus persicae em genótipos de tomateiro com altos teores foliares de aleloquímicos. Bragantia: 173-179. ZANIN, DS; RESENDE, JTV; ZEIST, AR; OLIVEIRA, JRF; HENSCHEL, JM; LIMA FILHO, RB. 2018. Selection of processing tomato genotypes resistant to two spotted spider mite. Horticultura Brasileira 36: 271-275. 